Onkologe 2015 · 21:198-200

DOI 10.1007/s00761-014-2835-7

Online publiziert: 13. Februar 2015

c) Springer-Verlag Berlin Heidelberg 2015

\author{
J.-U. Blohmer ${ }^{1}$ M. Krause ${ }^{2} \cdot$ K. Höffken ${ }^{3}$ P.M. Schlag ${ }^{4}$ \\ ${ }^{1}$ Charité - Universitätsmedizin Berlin \\ ${ }^{2}$ Technische Universität Dresden \\ ${ }^{3}$ Universitätsklinikum Düsseldorf \\ ${ }^{4}$ Berlin
}

\title{
Paradigma Brustkrebs
}

So wie bei den hämatologischen Neoplasien die akute Leukämie als Paradigma zur Erforschung von Pathogenese und Entwicklung von diagnostischen und therapeutischen Standards gilt, ist seit langer Zeit das Mammakarzinom paradigmatisch für die Fortschritte in Kenntnissen zur Grundlage sowie Versorgung von soliden Tumoren.

\section{》) Schrittmacherfunktion haben sowohl Grundlagenforschung als auch klinische Forschung}

Schrittmacherfunktion haben hier gleichermaßen sowohl Grundlagenforschung als auch klinische Forschung in Gestalt von großen interdisziplinären klinischen Studien.

Wenn man an die Zeit zurückdenkt, als das lymphogen metastasierte, primäre Mammakarzinom mit einer 85\%igen Wahrscheinlichkeit zu einem nur noch palliativ zu behandelnden Krankheitsbild wurde, so ist mit der heute zu verzeichnenden Prognose des frühen Mammakarzinoms als einer heilbaren Neoplasie so etwas wie ein Quantensprung zu verzeichnen.

Heute gibt es das primäre Mammakarzinom nicht mehr. Durch die Forschung und Entdeckung einer Vielzahl von genetischen Veränderungen neben der als epochal zu bezeichnenden Beschreibung der BRCA1- und BRCA2-Gene steht die Senologie vor der Möglichkeit einer individuell festzulegenden Prognose und einer genetisch begründbaren Therapie. Der Beitrag von R. Schmutzler gibt einen profunden Einblick in dieses wichtige Thema.

Bald wird eine risikoadaptierte Früherkennung das Massenscreening mit den zurecht kritisierten negativen Konse- quenzen abgelöst haben. I. Schreer gibt in ihrem Beitrag ihre langjährigen Erfahrungen und Kenntnisse an den Leser weiter.

Heute wird nicht mehr darüber gestritten, ob das primäre Mammakarzinom einer radikalen Mastektomie mit totaler axillärer Clearance zu unterziehen ist. Stattdessen zeigt J.-U. Blohmer, dass bei der standardmäßig durchzuführenden brusterhaltenden Operation die Genauigkeit der Resektionsrandbestimmung durchaus von Bedeutung ist, um eine optimale Tumorresektion im Gesunden zu erzielen, die Sentinel-Node-Diagnostik Begrenzungen durch die präoperative Therapie erfährt und Sofortrekonstruktionen einen festen Platz in der Primärbehandlung des frühen Mammakarzinoms eingenommen haben. All dies sind Themen, die früher durch die radikalen Therapiemaßnahmen überdeckt waren und denen keine Bedeutung zugemessen wurde.

Wo früher noch der Wert und die Art und Applikationszeit einer adjuvanten Chemotherapie getestet wurden, zeigt der kenntnisreiche Überblick von $M$. Untch und C. Thomssen, dass die neoadjuvante und adjuvante Chemotherapie eine zweifelsfreie Stellung in dem Gesamtbehandlungsplan des frühen Mammakarzinoms eingenommen haben und zu einer entscheidenden Prognoseverbesserung führen.

Die wechselnde Bedeutung der postoperativen Radiotherapie auf dem Weg zur heutigen Primärtherapie ist einer klaren Indikation zu Nachbestrahlung des brusterhaltend operierten Mammakarzinoms gewichen. Die heutige Richtung klinischer Forschung wird von M. Krause und K. Gurtner aus profunder Kenntnis der Materie beschrieben und mündet in der Feststellung, dass der heutige Therapiestandard der fraktionierten oder hypofraktionierten Ganzbrustbestrahlung mit 
Hier steht eine Anzeige.

黛 Springer 
anschließendem Boost möglicherweise schon bald im Sinne einer Deeskalation der Intensität durch Teilbrustbestrahlung oder Verzicht der Radiotherapie bei Niedrigrisikokonstellationen abgelöst wird.

Welche Schlüsse lassen sich nach dem Studium der Beiträge des aktualisierten, den neuesten Kenntnisstand wiedergebenden Leitthemas ziehen?

- Die Dynamik in der Forschung zur Pathogenese, Prognose und Therapie des frühen Mammakarzinoms ist ungebrochen hoch.

- Das Mammakarzinom ist unverändert als paradigmatisch für die klinische Forschung bei soliden Tumoren zu betrachten, da sich der Erkenntnisgewinn stetig in Erneuerungen der Therapiekonzepte umsetzt. Das Mammakarzinom ist das Paradigma translationaler Forschung.

- Das frühe Mammakarzinom ist zu einer heilbaren Erkrankung geworden.

Für die Schriftleiter

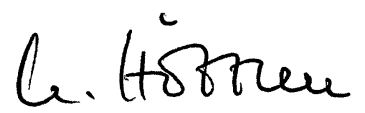

Klaus Höffken

für die Herausgeber

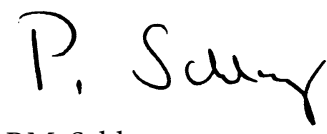

P.M. Schlag

\section{Korrespondenzadresse}

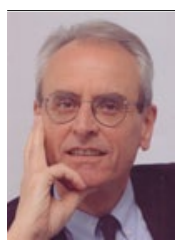

Prof. Dr. K. Höffken

Universitätsklinikum

Düsseldorf

Universitätsstraße 1

40225 Düsseldorf

Hoeffken.Editor@med.uni-

duesseldorf.de

\section{Einhaltung ethischer Richtlinien}

Interessenkonflikt. K. Höffken, J.-U. Blohmer, M. Krause und P.M. Schlag geben an, dass kein Interessenkonflikt besteht.

Der Beitrag enthält keine Studien an Menschen oder Tieren.

\section{Wandzellen der Blutgefäße steuern Metastasierung}

Wissenschaftler aus dem DKFZ und der Medizinischen Fakultät Mannheim der Universität Heidelberg suchten nach neuen Wegen, um bei Krebserkrankungen die Entstehung von Metastasen zu verhindern. Dazu kombinierten sie einen Antikörper gegen ein zentrales Steuerprotein der Blutgefäßzellen mit einer niedrigdosierten Chemotherapie. So behandelte Mäuse entwickelten weniger Metastasen und überlebten länger. Die Kombinationstherapie wirkt gleich mehrfach gegen eine Ansiedlung von Tochtergeschwülsten. Sie verhindert, dass Blutgefäße die neu entstehenden Metastasen versorgen. Gleichzeitig reduziert sie die Anzahl bestimmter Immunzellen, die die Ansiedlung von Krebszellen fördern.

Ausgangspunkt waren neue wissenschaftliche Erkenntnisse, die eine weitaus aktivere Rolle der Endothelzellen für das Tumorwachstum nahelegen, als dies bisher bekannt war. Ziel war es, nicht nur die Gefäßbildung in Tumoren zu unterdrücken, sondern gleichzeitig die Produktion dieser Wachstumsfaktoren zu hemmen. In ihrer aktuellen Publikation haben Srivastava et al. das Molekül Angiopoietin-2 ins Visier genommen. Es wird von Endothelzellen gebildet und spielt eine zentrale Rolle bei der Angiogenese.

Die Forscher übertrugen Brust- oder Lungenkrebszellen auf Mäuse, ließen Tumoren heranwachsen und entfernten sie zu einem frühen Zeitpunkt operativ. Um Metastasen vorzubeugen, erhielten die Tiere nach der Operation verschiedene Arten der Chemotherapie sowie teilweise zusätzlich einen blockierenden Antikörper gegen Angiopoietin-2. Während die Chemotherapie allein nicht wirksam war entwickelten mit dem Angiopoietin-2-Antikörper behandelte Tiere deutlich weniger Metastasen in Lunge und Knochen als ihre unbehandelten Artgenossen.

Gesteigert wurde dieser Effekt durch Kombination des Antikörpers mit einer so genannten metronomen Chemotherapie. Dabei werden die zytostatischen Substanzen niedrigdosiert dauerhaft verabreicht. Mäuse, die die Kombinationstherapie erhielten, lebten länger als Tiere, die nur mit dem Antikörper gegen Angiopoietin-2 behandelt wurden.
In anschließenden Gewebeanalysen untersuchten die Wissenschaftler, was die Kombinationstherapie genau bewirkt. Dabei entdeckten sie, dass Angiopoietin-2 nicht nur das Gefäßwachstum fördert, sondern darüber hinaus auf die Endothelzellen zurückwirkt und sie dazu anregt, tumorfördernde Makrophagen in die Umgebung der Krebszellen zu locken. Wurde Angiopoietin-2 blockiert, wanderten deutlich weniger krebsfördernde Immunzellen in die Tumorumgebung ein. Die niedrigdosierte metronome Chemotherapie, die die Wirksamkeit des Therapieansatzes weiter steigerte, richtet sich im Gegensatz zur herkömmlichen Hochdosischemotherapie nicht primär gegen die Tumorzellen selbst, sondern verhindert, dass sich bestimmte Zellen aus dem Knochenmark im Tumor ansiedeln, die ebenfalls das Tumorwachstum fördern.

"Mit unserer Kombinationstherapie gehen wir also von mehreren Seiten gleichzeitig gegen die Ansiedlung von Metastasen vor: Zum einen drosseln wir ihre Gefäßversorgung. Zum anderen verhindern wir, dass sich tumorfördernde Makrophagen ansiedeln, die eine entzündliche Umgebung schaffen und damit gewissermaßen den Boden für eine dauerhafte Ansiedlung der Krebszellen bereiten", erklärt Hellmut Augustin.

Dieses Wissen wollen die Forscher nun gezielt in eine klinische Anwendung übertragen.

Literatur

Srivastava K, Hu J, Korn C, Savant S, Teichert M, Kapel SS, Jugold M, Besemfelder E, Thomas M, Pasparakis M, Augustin HG (2014) Postsurgical adjuvant tumor therapy by combining anti-Angiopoietin-2 and metronomic chemotherapy limits metastatic growth. Cancer Cell 2014, DOI 10.1016/j.ccell.2014.11.005

Quelle: Oliver Meckes (Eye of Science)/ H. Augustin (DKFZ) 